\title{
MORFOMETRÍA GEOMÉTRICA Y FILOGENIA EN RHODNIINI (HEMIPTERA, REDUVIIDAE) DE VENEZUELA
}

\author{
Ana Soto-Vivas, ${ }^{1}$ Jonathan LiRiA ${ }^{2}$ \& Efraín De LunA ${ }^{3}$ \\ ${ }^{1}$ Servicio Autónomo Instituto de Altos de Estudios en Salud "Dr. Arnoldo Gabaldon", MPPSalud, \\ Aragua-Venezuela. \\ ${ }^{2}$ Departamento de Biología - FACYT, Universidad de Carabobo, Carabobo-Venezuela. \\ ${ }^{3}$ Biodiversidad y Sistemática - INECOL, Xalapa, Veracruz, México. \\ E-mail: anasoto.vivas@gmail.com; jliria@uc.edu.ve; efrain.deluna@inecol.edu.mx
}

Autor corresponsal: ANA SOTO-VIVAS. Centro de Estudios Enfermedades Endémicas y Salud

Ambiental. Servicio Autónomo Instituto de Altos Estudios en Salud "Doctor Arnoldo Gabaldon", Avenida Bermúdez Sur No 93, antiguo edificio de Malariología, Maracay, Estado Aragua, Venezuela; apartado postal 2171, 2113/ZIP 2101-A. Teléfono: (0058) 2432412846

Soto-Vivas, A., J. Liria \& E. De Luna. 2011. Morfometría geométrica y filogenia en Rhodniini (Hemiptera, Reduviidae) de Venezuela. Acta Zool. Mex. (n. s.), 27(1): 87-102.

RESUMEN. La tribu Rhodniini incluye los géneros Rhodnius Stål y Psammolestes Bergroth. Evidencias enzimática y molecular sugieren la monofilia de la tribu. La mayoría de las especies son selváticas encontrándose en palmas y en nidos de aves. Tradicionalmente ambos géneros fueron considerados grupos relacionados; no obstante, estudios moleculares cuestionan la monofilia de Rhodnius. El objetivo fue analizar filogenéticamente la variación morfométrica en la arquitectura alar, como apoyo a la taxonomía y sistemática en Rhodniini. Se fotografiaron 524 alas de cinco representantes de Rhodniini: Psammolestes arthuri (Pinto) $(n=89)$, Rhodnius pictipes Stål, $(n=21), R$. robustus Larrousse $(n=24)$, R. prolixus Stål, $(n=16)$ y $R$. neivai Lent $(n=22)$. Como grupos externos se estudiaron cuatro representantes de Triatomini: Eratyrus mucronatus Stål $(n=15)$, Panstrongylus rufotuberculatus (Champion) $(n=45)$, $P$. geniculatus (Latreille) $(n=183)$ y Triatoma maculata (Erichson) $(n=109)$. Se registraron configuraciones de coordenadas $(x, y)$ y se alinearon mediante Análisis Generalizado de Procrustes. Se efectuaron Análisis de Covarianza con proporción de grupos re-clasificados y MANOVA. Luego, las variables de la forma alar (intervalos de confianza de las deformaciones relativas) y el tamaño del centroide se analizaron cladísticamente. Los análisis estadísticos de varianza no encontraron diferencias significativas (Kruskal-Wallis) en el tamaño isométrico del ala en las especies $P$. arthuri-R. neivai-R. pictipes; $R$. robustus $-R$. prolixus $-T$. maculata y entre $P$. rufotuberculatus $-P$. geniculatus. La reclasificación $a$ posteriori fue perfecta para E. mucrunatus $100 \%$ y $R$. pictipes, seguidas de $T$. maculata con $96 \%, R$. neivai $95 \%$, P arthuri $93.2 \%$; R. prolixus $87.5 \%$, P. geniculatus $87.4 \%$, P. rufotuberculatus $84.4 \%$, y R. robustus $76 \%$. Los análisis cladísticos con parsimonia seleccionaron dos árboles de mínima longitud $(\mathrm{L}=4.461 \mathrm{IC}=0.973$ e IR $=0.979)$. El consenso estricto muestra la monofilia de Panstrongylus (rufotuberculatus + geniculatus) y Triatoma + Rhodniini (Rhodinus + Psammolestes), pero internamente la parafilia de Rhodnius respecto a Psammolestes. Estos resultados son congruentes con análisis molecu-

Recibido: 15/04/2010; aceptado: 22/09/2010. 
lares previos en Rhodniini, lo cual revela la información filogenética de caracteres morfométricos como apoyo a los estudios sistemáticos, permitiendo combinar por primera vez en este grupo los análisis de morfometría geométrica y métodos filogenéticos.

Palabras clave: Triatominae, Rhodnius, Psammolestes, morfometría, sistemática.

Soto-Vivas, A., J. Liria \& E. De Luna. 2011. Geometric morphometrics and phylogeny in Rhodniini (Hemiptera, Reduviidae) of Venezuela. Acta Zool. Mex. (n. s.), 27(1): 87-102.

ABSTRACT. Tribe Rhodniini includes Rhodnius Stål and Psammolestes Bergroth. Enzymatic and molecular evidence suggest the tribe is monophyletic. Most species are wild, living in palms and bird nests. Traditionally both genera were considered related; nevertheless, molecular studies don't support the Rhodnius monophyly. The goal was to phylogenetically analyze morphometric variation in wing architecture in support of Rhodniini taxonomy and systematics. We photographed 524 wings of five representatives of Rhodniini: Psammolestes arthuri (Pinto) $(n=89)$, Rhodnius pictipes Stål $(n=21)$, $R$. robustus Larrousse $(n=24)$, $R$. prolixus Stål $(n=16)$, and $R$. neivai Lent $(n=22)$. As outgroups we studied four representatives of Triatomini: Eratyrus mucronatus Stål $(n=15)$, Panstrongylus rufotuberculatus (Champion) $(n=45), P$. geniculatus (Latreille) $(n=183)$, and Triatoma maculata (Erichson) $(n=109)$. Landmark coordinate $(x, y)$ configurations were registered and aligned by Generalized Procrustes Analysis. Covariance Analyses were implemented with proportions of re-classified groups and MANOVA. Then, wing shape variables (confidence intervals from relative warps) and centroid size were cladistically analysed. Statistical analyses of variance found not significant differences in wing isometric size (Kruskal-Wallis) among $P$. arthuri-R. neivai-R. pictipes; $R$. robustus $-R$. prolixus- $T$. maculata and between $P$. rufotuberculatus $-P$. geniculatus. The a posteriori re-classification was perfect in E. mucrunatus $100 \%$ and $R$. pictipes, followed by $T$. maculata $96 \%, R$. neivai $95 \%, P$ arthuri $93.2 \%$; R. prolixus $87.5 \%$, P. geniculatus $87.4 \%$, P. rufotuberculatus $84.4 \%$, and $R$. robustus $76 \%$. Cladistic analyses under parsimony selected two most parsimonious trees $(\mathrm{L}=4.461 \mathrm{IC}=0.973$ and $\mathrm{IR}=0.979)$, where the strict consensus showed a monophyletic group with Panstrongylus (rufotuberculatus + geniculatus) and Triatoma + Rhodniini (Rhodinus + Psammolestes), but internally it shows the paraphyly of Rhodnius regarding Psammolestes. The congruence between these results and previous molecular analyses in Rhodniini, reveal the phylogenetic information of our morphometric characters as support to systematic studies, allowing the combination of geometric morphometrics and phylogenetic methods for the first time in this group.

Key words: Triatominae, Rhodnius, Psammolestes, geometric morphometrics, systematics, cladistic analyses.

\section{INTRODUCCIÓN}

Triatominae se clasifica como subfamilia de Reduviidae (Hemiptera, Heteroptera) y se define por su hábito hematófago y adaptaciones morfológicas asociadas a la alimentación con sangre. Hasta la fecha se han descrito 140 especies (Schofield \& Galvão 2009). Los triatóminos son vectores de Trypanosoma cruzi (Chagas 1909) agente causal de la tripanosomiasis americana o enfermedad de Chagas, una de las parasitosis de mayor importancia médica en América Latina (Lent \& Wygodzinski 1979, World Bank 1993). La taxonomía de los triatóminos ha sido estudiada por varios autores examinando la variación de fuentes de evidencia morfológica y molecular bajo distintos enfoques analíticos (Dujardin et al. 1997, 1988, Lyman, et al. 
1999, Hypša et al. 2002, Schofield \& Galvão, 2009). No obstante, la clasificación de las especies de Rhodnius, que incluye importantes especies transmisoras de tripanosomiasis, todavía es poco robusta. Este trabajo presenta nueva evidencia de los patrones ínter específicos de variación alar, la cual se interpreta con ayuda de análisis de morfometría geométrica y análisis filogenéticos. Estos análisis combinados permiten por primera vez resaltar la importancia de la forma de las alas en la clasificación e identificación de especies de Rhodnius.

La subfamilia Triatominae está conformada por cinco tribus, definidas por caracteres relacionados a la hematofagia. Especialmente las tribus Triatomini y Rhodniini presentan marcada disparidad morfológica y adaptaciones fisiológicas, por ejemplo al nivel de glándulas salivares (Schofield 1988, 1996, Catalá 1997, Ribeiro et al. 1998). La tribu Rhodniini incluye los géneros Rhodnius Stål, 1859 y Psammolestes Bergroth, 1911. Evidencia enzimática y molecular sugieren la monofilia de la tribu (Lent \& Wygodzinsky 1979, Schofield 1988, 1994, Monteiro et al. 2000, Tartarotti et al. 2006). Pero, estudios recientes han cuestionado la monofilia de Rhodniini (Lyman et al. 1999; Monteiro et al. 2000; Hypša et al. 2002). La mayoría de las especies de la tribu son de hábitats selváticos viviendo en las palmas y en los nidos de las aves. Solo algunas especies de Rhodnius han logrado colonizar las viviendas humanas, por lo cual son consideradas con gran importancia vectorial; tal es el caso de Rhodnius prolixus Stål, 1859 el vector principal de la enfermedad de Chagas en Venezuela, Colombia y parte de Centro América.

El género Psammolestes se compone de tres especies: P. coreodes Bergroth, 1911, $P$. arthuri Pinto, 1926 y P. tertius Lent \& Jurberg, 1965 (Galvão et al. 2003). Se sugiere que estas especies se especializaron para explotar microhábitats como nidos de aves en bosques abiertos (Lent \& Wygodzinsky 1979,Abad-Franch et al. 2009). P. arthuri es el representante más septentrional de Psammolestes, ampliamente distribuido en Venezuela y Colombia (Carcavallo et al. 1999, 2000, Gurgel-Gonçalves \& Bandeira 2009); se asocia principalmente con el peridomicilio (Lent \& Jurberg 1965) en los Llanos de Venezuela y en la Provincia biogeográfica de Costa de Venezuela (Abad-Franch \& Monteiro 2007). Según la bibliografía $P$. arthuri es la única especie del género Psammolestes presente en Venezuela.

Dentro del género Rhodnius se clasifican 16 especies y se han propuesto dos grupos de difícil identificación dado a su similitud morfológica. El primero es el "complejo prolixus" formado por cinco especies ( $R$. prolixus, $R$. robustus Larrousse, $1927, R$. neglectus Lent, $1954, R$. domesticus Neiva y Pinto, 1923 y $R$. nasutus Stål, 1859). El segundo es el "complejo pallescens" formado por ocho especies $(R$. pallescens, $R$. colombiensis Mejia, Galvão \& Jurberg, 1999, R. ecuadoriensis Lent \& León, 1958, R. paraensis Sherlock, Guitton \& Miles, 1977, R.. pictipes Stål, 1872, R. dalessandroi Carcavallo \& Barreto, 1976, R. brethesi Matta, 1919 y R. stali Lent, Jurberg \& Galvão, 1993). Para las otras tres especies no se han encontrado elementos 
morfológicos y moleculares que las ubiquen (Lent \& Wygodzinski 1979, Dujardin et al. 1999, Schofield \& Dujardin 1999). Dentro del "complejo proxilus" algunos autores sugieren que $R$. robustus es la variante selvática y $R$. prolixus es derivada de las poblaciones domiciliadas (Harry et al. 1992; Harry 1993a, b; Barrett 1995, Schofield \& Dujardin 1999). El género Rhodnius esta representado en Venezuela por seis especies: $R$. robustus, $R$. prolixus, $R$. pictipes, $R$. neivai, $R$. brethesi y $R$. pallescens (Cova \& Suárez 1959, Lent \& Wygodzinsky 1979, Torrealba et al. 1985, Osuna 1984, Carcavallo et al. 1999, Galvão et al. 2003, Schofield \& Galvão 2009, Soto-Vivas 2009).

La morfometría geométrica (Bookstein 1991, Rohlf \& Marcus 1993, Adams et al. 2004, Zieldich et al. 2004) ha sido utilizada en insectos de importancia médica como flebotominos, mosquitos y triatóminos, para diferenciar poblaciones domiciliadas y silvestres, en estudios ontogéneticos (Dujardin et al. 1997, 1998, Feliciangeli et al. 2007, Soto-Vivas et al. 2007, Gonacalves 2008), análisis de variaciones altitudinales (Belen et al. 2004), para detectar diferencias en poblaciones de campo y colonia (Jaramillo et al. 2002), o entre diferentes regiones geográficas (Monroy et al. 2003, Lehmann et al. 2005, Yurtas et al. 2005), e inclusive como herramienta taxonómica en la discriminación de especies (Matias et al. 2001, Calle et al. 2002, 2008). Las herramientas morfométricas han demostrado su utilidad en la discriminación de insectos vectores que no pueden identificarse por medio de caracteres morfológicos tradicionales, así como en estudios de variabilidad intra-específica (Dujardin et al. 1997, 1998, Rubio-Pallis 1998, Calle et al. 2002, 2008, Yurtas et al. 2005). Los datos morfométricos también han sido empleados para la postulación de hipótesis cladísticas, demostrando que estos caracteres contribuyen en el apoyo de grupos monofiléticos (Guerrero et al. 2003, Acero et al. 2005, Camul \& Polly 2005, Bogdanowicz et. al. 2005, Goloboff et al. 2006, González-José et al. 2008, de Bivort et al. 2010). Más recientemente, Catalano et al. (2010) han desarrollado algoritmos para realizar análisis cladísticos a partir de la inclusión directa sin codificación de las coordenadas $x, y$ (y 3D) de especímenes alineados.

Como parte de un estudio taxonómico y biogeográfico de los Triatominae de Venezuela, el presente trabajo utiliza herramientas de la morfometría geométrica en combinación con análisis cladísticos con el fin de estudiar la variación de la arquitectura alar, determinando diferencias y cambios en tamaño y conformación, como apoyo a la taxonomía y sistemática de las especies de la tribu Rhodniini.

\section{MATERIAL Y MÉTODOS}

Adquisición de datos. Se revisó material depositado en siete colecciones entomológicas: Museo Cova-García, del Instituto de Altos Estudios en Salud Pública "Dr. Arnoldo Gabaldon"; Museo de Zoología Agrícola, Fac. de Agronomía, Universidad 
Central de Venezuela (MIZA), Museo Entomológico "José M. Osorio", Universidad Centro Occidental de los Llanos (UCLA); Colección Herman Lent, Universidad de los Andes (ULA); Colección de Artrópodos, Universidad del Zulia (LUZ); Colección de Entomología, Universidad Experimental Francisco de Miranda; Colección privada Familia Romero (FR). Se fotografiaron digitalmente las alas de 524 ejemplares de cinco especies de Rhodniini ( $P$. arthuri $(n=89), R$. pictipes $(n=21), R$. robustus $(n=$ $24), R$. prolixus $(n=16)$ y $R$. neivai $(n=22))$ y cuatro especies de Triatomini (Eratyrus mucronatus Stål, 1859 ( $n=15)$, Panstrongylus rufotuberculatus (Champion, 1899) $(n=45), P$. geniculatus (Latreille, 1811) $(n=183)$ y Triatoma maculata (Erichson, $1848(n=109))$. En cada ala se ubicaron seis puntos anatómicos de referencia (PAR 1-6), los cuales corresponden con el tipo I sensu Bookstein (1991): Base de la vena transversal cu-pcu (PAR1), Base de la vena transversal m-cu (PAR2), doblez de la vena $\mathrm{m}$ (PAR3), intercepción de la vena $\mathrm{R}+\mathrm{Sc}$ (PAR4), Ápice de la vena M (PAR5) y ápice de la intercepción cu-Pcu (PAR6). Los PAR 1-4 se ubican en yuxtaposición con el área coriácea del ala. La configuración de los seis pares de coordenadas $x y$ de cada ala (Fig. 1) se capturó mediante la ayuda del programa TPSDig (Rohlf 2007).

Análisis de morfometría geométrica. A partir de la matriz de 524 configuraciones de coordenadas geométricas de los seis puntos (PAR1-6) se realizó el Análisis

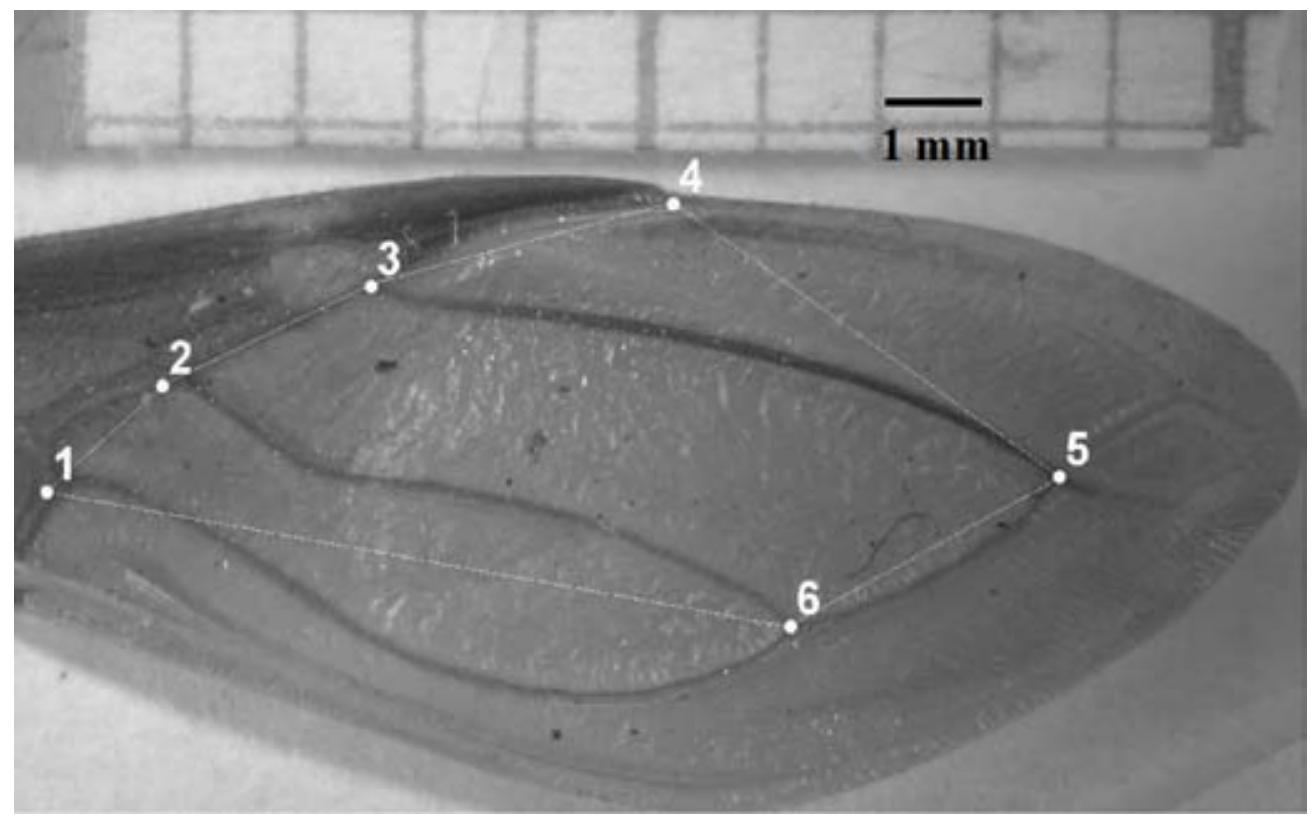

Figura 1. Microfotografía del ala derecha de Eratyrus mucronatus mostrando la disposición de los puntos anatómicos de referencia (PAR 1-6). 
Generalizado de Procrustes, con el programa CoordGen (Sheets 2005a) para una superimposición Procrustes y luego extraer una matriz con las variables de conformación (partial warps $=\mathrm{Pw}$ ) y el tamaño centroide $(\mathrm{CS})$. Esta matriz de Pw se usó para un Análisis de Variables Canónicas con el programa CVAGen (Sheets 2005b), mediante el cual también se obtuvo la proporción de grupos (= especies) re-clasificados y MANOVA por cada eje canónico. Las diferencias en los valores de CS entre las especies fueron analizadas mediante la prueba de Kruskall-Wallis $(P 0,05)$. Luego se calcularon las deformaciones relativas respecto a la configuración consenso (relative warps o Rw) con $\alpha=0$ en el programa TpsRelw (Rolhf 2003) para su inclusión como caracteres continuos en los análisis filogenéticos.

Análisis cladístico. La selección de árboles óptimos fue realizada con el programa de parsimonia TNT 1.1 (Goloboff et al. 2008), el cual permite el uso de caracteres con valores continuos para obtener hipótesis filogenéticas. Se construyó una matriz con los rangos de los valores de Rw y CS para cada especie, a partir de intervalos de confianza $(\mathrm{CI}=\mathrm{Media} \pm \mathrm{Z} \alpha / 2 \cdot \mathrm{S} / \sqrt{\mathrm{N}})$ con $\alpha=0.05$ (Norman \& Streiner, 2000). Se siguió el método propuesto por Goloboff et al. (2006), en donde los caracteres continuos, Rw y CS en este caso, son tratados como aditivos. Los rangos son optimizados directamente en los árboles, utilizando el algoritmo de Farris (1970) para el down-pass y Goloboff (1993) para el up-pass. Se consideraron las cuatro especies de Triatomini como grupos externos y E. mucronatus se especificó para enraizar los cladogramas. Se empleó el algoritmo de enumeración implícita para la búsqueda de las hipótesis más parsimoniosas. Se estimó el soporte de clados mediante técnicas de remuestreo por Jackknifing (Farris et al. 1996) y Bootstrapping (Felsenstein 1985), con 1000 réplicas y búsquedas también por enumeración implícita. Finalmente se estimaron los cambios de conformación del ala a partir de la optimización de coordenadas $x, y$ en el árbol de consenso estricto.

\section{RESULTADOS}

Tamaño isométrico. No se encontraron diferencias significativas (Kruskal-Wallis: $\left.\chi^{2}=443.49 p<0.001\right)$ en el tamaño isométrico del ala (Cuadro 1) en las especies $P$. arthuri $(5.776 \pm 0.037)-R$. neivai $(5.764 \pm 0.125)-R$. pictipes $(6.176 \pm 0.113) ; R$. robustus $(7.829 \pm 0.226)-R$. prolixus $(7.525 \pm 0.194)-T$. maculata $(7.272 \pm 0.063)$; $P$. rufotuberculatus $(9.853 \pm 0.088)-P$. geniculatus $(10.029 \pm 0.059)$.

CVA y cambio de la forma del ala. En el Cuadro 2 se presentan los resultados de la prueba de re-clasificación en los siete ejes discriminantes del CVA con los valores del Wilks lambda, $\chi 2$, grados de libertad y probabilidades. La clasificación de las 524 muestras en los grupos a priori fue basada según la identificación de las especies por la morfología tradicional y la re-clasificación a posteriori depende de las distancias de Mahalanobis entre cada muestra y la media de cada especie. Las muestras re-cla- 
Cuadro 1. Promedio y error estándar del estimador de tamaño isométrico (en mm) para las especies de Triatominae.

\begin{tabular}{clccc}
\hline \multicolumn{1}{c}{ Especies } & $\begin{array}{c}\text { Tamaño } \\
\text { promedio }\end{array}$ & Centroide & $\begin{array}{c}\text { Error } \\
\text { estándar }\end{array}$ \\
\hline A & Psammolestes arthuri & 5.7766 & AF & 0.0378 \\
B & Panstrongylus rufotuberculatus & 9.8530 & BD & 0.0881 \\
C & Eratyrus mucronatus & 8.4861 & EC & 0.1665 \\
D & Panstrongylus geniculatus & 10.0293 & BD & 0.0596 \\
E & Rhodnius robustus & 7.8292 & CE & 0.2262 \\
F & Rhodnius neivai & 5.7643 & FA & 0.1254 \\
G & Rhodnius pictipes & 6.1760 & AG & 0.1130 \\
H & Rhodnius prolixus & 7.5235 & CEI & 0.1946 \\
I & Triatoma maculata & 7.2721 & EH & 0.0633 \\
\hline
\end{tabular}

Kruskal-Wallis: $\chi^{2}=443.49 p<0.001$. Letras indican las especies donde no hay diferencias significativas del tamaño centroide.

Cuadro 2. Clasificación para los 524 especimenes de Triatominae y valores de lambda $(\Lambda)$ de Wilk's para cada eje canónico.

\begin{tabular}{lcccccccccc}
\hline \multicolumn{1}{c}{ Asignaciones a priori } & \multicolumn{7}{c}{ Asignaciones a posteriori } \\
\hline Psammolestes arthuri & 83 & 3 & 0 & 0 & 0 & 0 & 0 & 3 & 0 \\
Panstrongylus rufotuberculatus & 0 & 38 & 1 & 4 & 0 & 0 & 0 & 0 & 2 \\
Eratyrus mucronatus & 0 & 0 & 15 & 0 & 0 & 0 & 0 & 0 & 0 \\
Panstrongylus geniculatus & 0 & 19 & 4 & 160 & 0 & 0 & 0 & 0 & 0 \\
Rhodnius robustus & 0 & 0 & 0 & 0 & 19 & 2 & 0 & 4 & 0 \\
Rhodnius neivai & 0 & 0 & 0 & 0 & 0 & 19 & 2 & 0 & 0 \\
Rhodnius pictipes & 0 & 0 & 0 & 0 & 0 & 0 & 21 & 0 & 0 \\
Rhodnius prolixus & 0 & 0 & 0 & 0 & 2 & 0 & 0 & 14 & 0 \\
Triatoma maculata & 0 & 1 & 1 & 0 & 1 & 0 & 1 & 0 & 105 \\
\hline
\end{tabular}

$\Lambda^{1}=0.0039 \chi^{2}=2854.5337 \mathrm{df}=64 p<0.001 ; \Lambda^{2}=0.0325 \chi^{2}=1763.6563 \mathrm{df}=49 p<0.001 ; \Lambda^{3}=0.1887 \chi^{2}=$ $858.0487 \mathrm{df}=36 p<0.001 ; \Lambda^{4}=0.5369 \chi^{2}=319.9466 \mathrm{df}=25 p<0.001 ; \Lambda^{5}=0.7605 \chi^{2}=140.8341 \mathrm{df}=16 p<$ $0.001 ; \Lambda^{6}=0.9017 \chi^{2}=53.2359 \mathrm{df}=9 p<0.001 ; \Lambda^{7}=0.9670 \chi^{2}=17.2582 \mathrm{df}=4 p=0.001$.

sificadas perfectamente al $100 \%$ fueron las de E. mucronatus $(15 / 15)$ y $R$. pictipes (21/21), seguidas de T. maculata con $96 \%$ de las muestras (105/109) y R. neivai $95 \%$ (19/21), P arthuri 93.2\% (83/89). Las especies con porcentaje de reclasificación inferior fueron $R$. prolixus $87.5 \%$ (14/16), P. geniculatus $87.4 \%$ (160/183), P. rufotuberculatus con $84.4 \%$ (38/45) y $R$. robustus $76 \%(19 / 25)$.

Visualización de los cambios de la forma alar sobre el espacio del CVA. La función de interpolación de placas delgadas (thin plate spline, TPS) permitió infe- 
rir las diferencias en conformación como deformaciones de los seis puntos en una rejilla calculada con respecto a la configuración consenso (Fig. 2). En general, la ubicación de los PAR 5 y 6 en el CVA1 no mostraron diferencias importantes entre los grupos de especies. Sin embargo, las especies de la Tribu Triatomini mostraron desplazamientos de los PAR 1-4. La base de la vena transversal cu-pcu muestra un desplazamiento hacia el área basal del ala; los puntos 2 y 3 se desplazan hacia arriba, ocasionando un ensanchamiento en la celda alar correspondiente a las venas cu y $\mathrm{M}$. En cambio, la celda alar en T. maculata se estrecha, pues los mismos PAR 1-4 se desplazan en sentido contrario al resto de las especies de la Tribu.

Las especies pertenecientes a la Tribu Rhodniini, mostraron un desplazamiento de todos los PAR. La base de la vena transversal cu-pcu se mueve hacia el área coriácea del ala, mientras que la base de la vena transversal m-cu y la vena M y la intercepción R-Scu muestran un desplazamiento hacia el interior del ala; así mismo el ápice de la vena $\mathrm{M}$ se mueve diagonalmente hacia arriba, y el ápice de la intercepción cu-Pcu se introduce lateralmente hacia el ala, en consecuencia el corium del ala de las especies

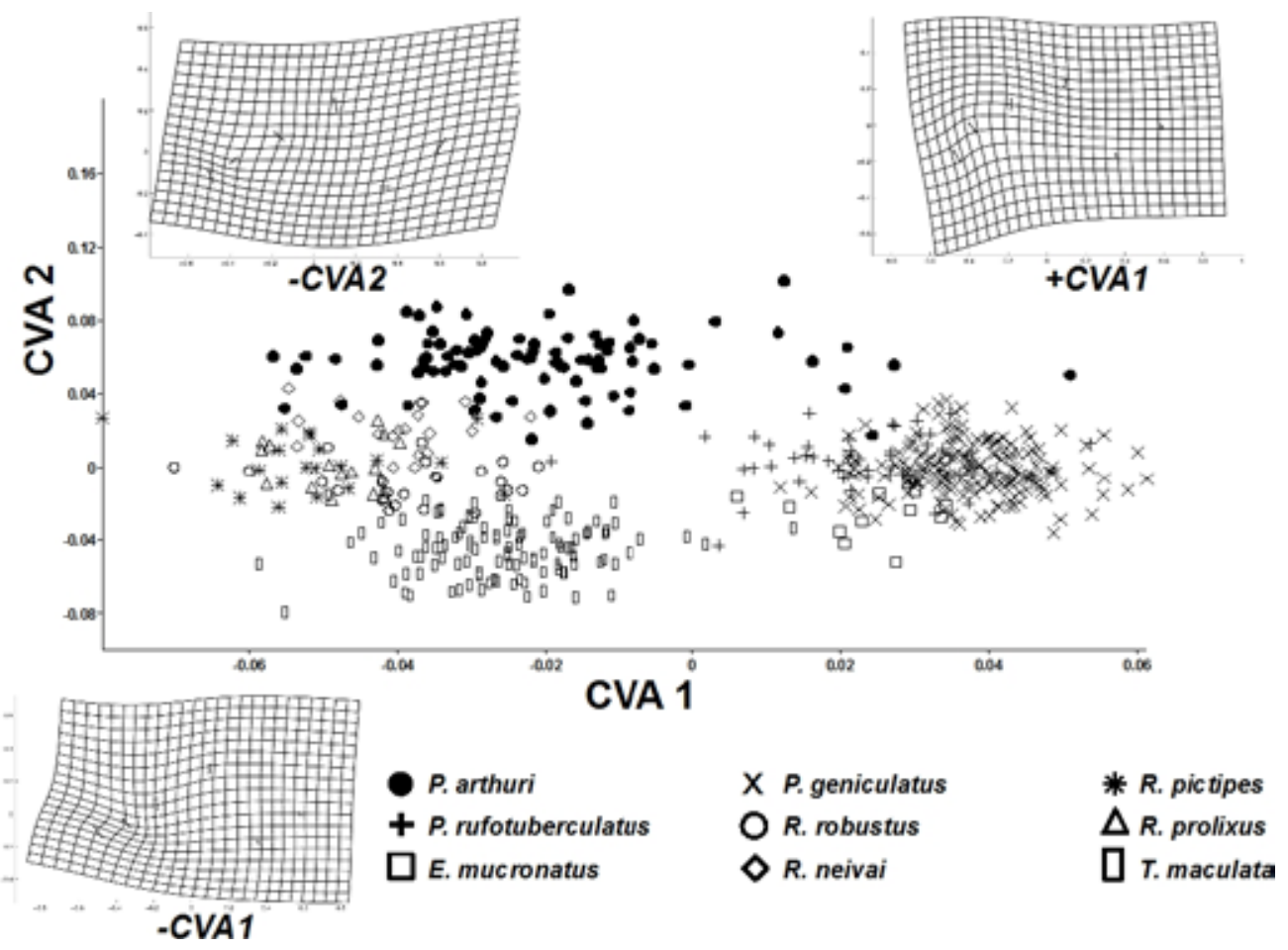

Figura 2. Diagrama de los dos primeros factores canónicos del CVA para los 524 especímenes de Triatominae, mostrando rejillas de deformación en las conformaciones extremas de los ejes. 
de la Tribu Rhodniini se desplaza hacia el área basal del ala, mientras que la región membranosa se estrecha y alarga.

Análisis cladístico. Se obtuvieron dos árboles de mínima longitud $(\mathrm{L}=4.461$ $\mathrm{IC}=0.973$ e IR $=0.979$ ). El consenso estricto de estos (Fig. 3) muestra tres clados relevantes: A, B y C. El clado C incluye las dos especies de Panstrongylus estudiadas (Jackknifing 76 / boostraping 79). Las sinapomorfías para este grupo son: Relative warp2 (carácter 1): 0.003-0.024 $\rightarrow-0.019-0.016$ y CS (carácter 8): 8.159-8.812 $\rightarrow$

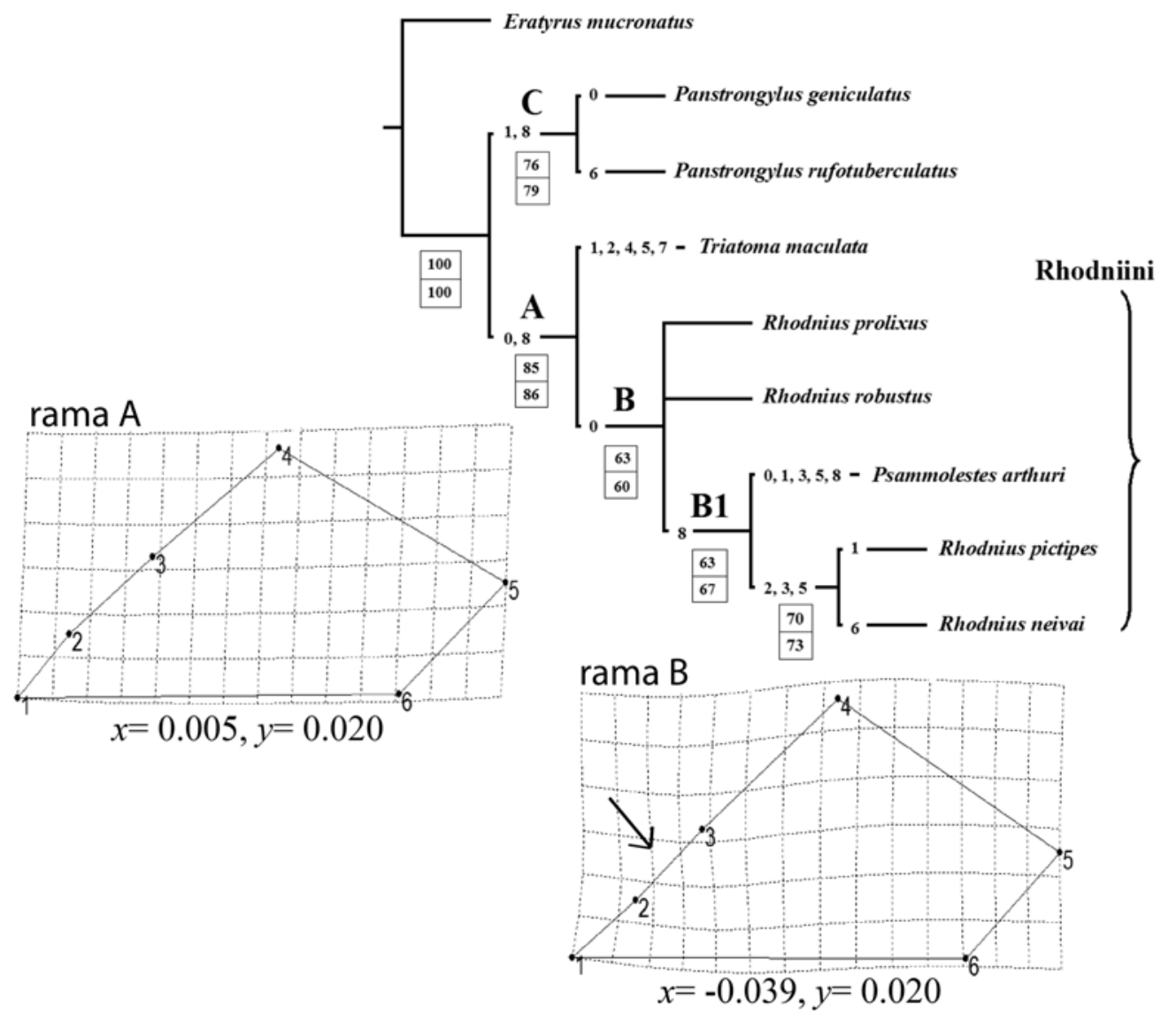

Figura 3. Consenso estricto de los dos árboles más parsimoniosos. Este árbol representa la mejor hipótesis de agrupamientos de las especies de Rhodniini según los análisis filogenéticos de los datos morfométricos de la forma ( $\mathrm{Pw}$ ) y tamaño alar (CS). Los clados relevantes en este estudio, A, B, C, son los mismos que se discuten en el texto. Los números sobre las ramas $(0$ al 8$)$ son las sinapomorfías comunes en los dos árboles. Los números en las cajas bajo las ramas (60 al 100) son los índices de soporte de grupos (Jacknifing/Bootstraping). Las rejillas de deformaciones ilustran las conformaciones de las alas reconstruidas en los nodos A y B. 
9.680-9.912. El clado A (85/86), agrupa a Triatoma maculata + Rhodniini por la presencia de las sinapomorfias: Relative warp 1 (caracter 0): 0.025-0.028 $\rightarrow$ 0.002-0.008 (Fig. 3A) y CS: 8.159-8.812 $\rightarrow$ 7.385-7.396. En el clado B se incluyen las especies de Rhodniini (62/60), soportado por una sinapomorfía el Relative warp 1 (carácter 0 ): 0.002-0.008 $\rightarrow-0.041-0.037$ (Fig. 3B). Luego se aprecia una tricotomía con Rhodnius prolixus, Rhodnius robustus y el clado B1 (63/67) que agrupa a Psammolestes arthuri + Rhodnius pictipes + Rhodnius neivai soportado por una sinapomorfía en CS: 7.385-7.396 $\rightarrow$ 5.954-6.010. Finalmente algunas especies mostraron caracteres autapomórficos: P. arthuri con Relative warp $1:-0.049-0.040 \rightarrow-0.062-0.051$, Relative warp 2: 0.016-0.024 $\rightarrow-0.039-0.032$, Relative warp4 (carácter 3): -0.003$0.001 \rightarrow 0.016-0.012$, Relative warp6 (carácter. 5): $-0.010-0.003 \rightarrow 0.005-0.012$ y CS: $5.954-6.010 \rightarrow 5.702-5.850, P$. rufotuberculatus con Relative warp7 (carácter 6): $-0.001-0.001 \rightarrow-0.018-0.013, P$. geniculatus con Relative warp $1:$ 0.025-0.028 $\rightarrow$ 0.031-0.035, $R$. neivai con Relative warp $7:-0.001-0.001 \rightarrow-0.022-0.014, R$. pictipes con Relative warp $1: 0.016-0.024 \rightarrow 0.033-0.049$, y Triatoma maculata con Relative warp 1: 0.016-0.024 $\rightarrow$ 0.045-0.052, Relative warp3 (caracter 2): -0.005 $0.001 \rightarrow-0.015-0.008$, Relative warp5 (caracter. 4): $-0.006-0.000 \rightarrow 0.002-0.008$, Relative warp6: $-0.005-0.003 \rightarrow 0.008-0.012$ y Relative warp8 (caracter 7): 0.000 $\rightarrow-0.004-0.001$.

Se llega a la misma hipótesis de filogenia de Rhodniini (resultados no incluidos) a partir de análisis filogenéticos obtenidos con diferentes métodos (Maximun Likelihood, Felsenstein 2002) o con diferentes variables de la forma (coordenadas $x y$ de los puntos anatómicos) analizadas en TNT según procedimientos filogenéticos propuestos recientemente (Catalano et al. 2010; Goloboff \& Catalano 2010).

Visualización de los cambios de la forma alar sobre la filogenia. Mediante la optimización de los caracteres continuos (Relative warps + tamaño centroide) en el árbol de consenso estricto se obtuvieron rangos de las sinapomorfías para cada nodo, lo cual permitió ingresar el valor medio en tpsRelw para reconstruir las conformaciones alares. En el caso del nodo A (Fig. 3A), la configuración de coordenadas muestra un desplazamiento horizontal de la intercepción de las venas R-Scu (PAR4) y el ápice de la vena M (PAR5), mientras que en el nodo B (Fig. 3B) se aprecian deformaciones diagonales en la base de la vena transversal m-cu (PAR2), dobles de la vena $\mathrm{M}$ (PAR3) y ápice de la intercepción de las venas cu-Pcu (PAR6).

\section{DISCUSIÓN}

Tamaño del ala. Los resultados de los análisis de la variación de tamaño de las alas, estimado por el tamaño del centroide (CS), revelaron diferencias significativas entre especies. Falconer (1981) propone que los caracteres métricos son los primeros que cambian en el transcurso de la evolución y esta responde al compromiso de la demanda del entorno y las del genoma. En este sentido, la variación en tamaño del ala 
detectada puede estar asociada a diferentes ambientes o a diferentes modos de vida y ser el resultado de causas macro y micro evolutivas. Las especies pequeñas explotan ecotopos como palmas y nidos de aves, tal es el caso de $P$. arthuri asociado a nidos de Phascellodomus rufifrons y otros Fornaridae, mientras que $R$. neivai se asocia a las palmeras (Copernitia tectorum) y troncos de árboles secos (Carcavallo et al. 1998). En particular resalta el hecho que en este estudio no se evidenciaron diferencias en tamaño entre $R$. robustus y $R$. prolixus. Estas especies ocupan ecotopos como palmeras silvestres (Longa y Scorza 2005) y algunos autores han sugerido que $R$. robustus es la forma selvática y $R$. prolixus se originó como adaptaciones al domicilio.

La visualización de las diferencias de conformación. La función de placas delgadas (thin plate spline) que se interpola en el AGP permitió observar las diferencias de conformación como deformaciones de rejillas con respecto a una configuración consenso. Las diferencias de conformación observadas entre las especies fueron profundas a excepción de las especies del complejo prolixus, permitiendo a los análisis discriminantes hacer reclasificaciones muy buenas. Este patrón de cambio del ala es congruente con la hipótesis de la divergencia evolutiva que probablemente comenzó al inicio de la separación de las especies de Rhodniini (Schofield \& Dujardin 1999).

Filogenia. Rhodniini se ha propuesto como un grupo monofilético (Lent \& Wygodzinski 1979) que incluye los géneros Rhodnius y Psammolestes. Esta clasificación se basa en características no compartidas por otros triatóminos, como la inserción apical de la antena, la forma corporal, callosidades postoculares, genitales masculinos, arquitectura superficial de los huevos y la presencia de nitroforina en las glándulas salivales (Lent \& Wygodzinsky 1979, Tartarotti et al. 2006). Sin embargo, análisis filogenéticos con base en ARN ribosómico, Citocromo B y la región 28S ARN nuclear (D2) incluyen a Psammolestes con especies de Rhodnius (Lyman et al. 1999; Monteiro et al. 2000). Particularmente, se detectó un clado que contempla a Psammolestes tertius y $R$. pictipes $+R$. brethes $+R$. ecuatoriensis $+R$. pallescens. Otro clado propuesto es el formado por especies del grupo prolixus ( $R$. nasutus $+R$. neglectus $+R$. domesticus $+R$. prolixus $+R$. robustus) y $R$. neivai (Monteiro et al. 2000). Recientemente, Hypša et al. (2002) también encontraron que Rhodniini es monofilético y que Rhodnius es parafilético respecto a Psammolestes según su análisis filogenético de 57 especies de tres tribus de Triatominae basado en secuencias de regiones del 16S y $12 \mathrm{~S}$ del ADN ribosomal.

Los resultados cladísticos y la hipótesis de parafilia en Rhodnius del presente trabajo son congruentes con los cambios taxonómicos señalados por Hypša et al. (2002: 456), en donde las especies de Psammolestes pasarían a formar parte de Rhodnius en nueva combinación genérica: Rhodnius arthuri (Pinto, 1926) comb. n., $R$. coreodes (Bergroth, 1911) comb. n., y R. tertius (Lent \& Jurberg, 1965). La parafilia de Rhodnius soportada tanto con datos moleculares (Hypša et al. 2002; Lyman et al. 1999; Monteiro et al. 2000) como con nuestros datos morfométricos analizados filogenéti- 
camente indican que la tribu Rhodniini diversificó rápidamente con grandes cambios morfológicos, entre ellos la pérdida de los caracteres ancestrales compartidos por los miembros de este grupo monofilético.

En conclusión, nuestros resultados revelan la importancia de los caracteres morfométricos como apoyo a los estudios sistemáticos en Triatominae. Esta investigación combina por primera vez el análisis de puntos anatómicos mediante morfometría geométrica y los métodos filogenéticos utilizando caracteres morfométricos continuos. Este estudio hace una comparación entre dos maneras de interpretar cambio de forma. La pregunta de investigación es la misma: ¿cuál es el cambio de forma? La respuesta usual en todo análisis de morfometría es usar el plano de la ordenación del CVA (ordenación multivariada sin jerarquía) para inferir el cambio de forma. La respuesta complementaria que ilustra este trabajo es usar el cladograma (ordenación multivariada con jerarquía) para inferir el cambio histórico de la forma. Este trabajo documenta que la estimación del cambio de forma es más apropiada para la taxonomía de un grupo cuando se le da el enfoque filogenético, para el análisis de las configuraciones de coordenadas después de la superposición Procrustes y el cálculo de las deformaciones relativas.

AGRADECIMIENTOS. Este trabajo se realizó con el apoyo de las siguientes personas e Instituciones que permitieron revisar los ejemplares: Sra. Hortencia Frontado asistente, Museo Cova-García IAES "Dr. Arnoldo Gabaldon"; Dr. Eduardo Osuna, Curador MIZA-UCV; Dra. Rosa Briseño, Curadora Museo Entomológico “José M. Osorio" UCLA; Dr. Elis Aldana, Curador Colección Herman Lent ULA; Ing. Agr. José Camacho, curador Colección de Artrópodos LUZ; Ing. Agr. Belkis Gómez, curadora Colección Entomológica, UEFM; Sr. Francisco Romero curador, Colección Privada Familia Romero.

\section{LITERATURA CITADA}

Abad-Franch F. \& F. A. Monteiro. 2007. Biogeography and evolution of Amazonian triatomines (Heteroptera: Reduviidae): implications for Chagas disease surveillance in humid forest ecoregions. Memorias do Instituto Oswaldo Cruz, 102 (Suppl 1): 57-70.

Abad-Franch F., Monteiro F. A., Jaramillo N., Gurgel.-Gonçalves R., Dias F. B. \& L. Diotauiti. 2009. Ecology, evolution, and the long-term surveillance of vector-borne Chagas disease: A multiscale appraisal of the tribe Rhodniini (Triatominae). Acta Tropica, 110(2-3): 159-167.

Acero A., Tavera, J. J. \& J. Reyes. 2005. Systematics of the genus Bagre (Siluriformes: Ariidae): A morphometric approach. Cybium, 29(2): 127-133.

Adams D. C., Rolhf F. J. \& D. E. Slice. 2004. Geometric morphometrics: Ten years of progress following the 'revolution'. Italian Journal of Zoology, 71: 5-16.

Barrett T. 1995. Species interfertility and crossing experiments in Triatomine systematics. In: Proceedings of the International Workshop on Population Genetics and Control of Triatominae, pp 72-77.

Belen A. Alten, B. \& A. M. Aytekin. 2004. Altitudinal variation in morphometric and molecular characteristics of Phlebotomus papatasi populations. Medical and Veterinary Entomology, 18: 343-350. 
Bogdanowicz W., Juste J., Owen R. D. \& A. Sztencel. 2005. Geometric morphometrics and cladistics: testing evolutionary relationships in mega- and microbats. Acta Chiropterologica, 7(1): 39-49.

Bookstein F. L. 1991. Morphometric tools for landmark data: Geometry and biology. New York: Cambridge University Press. 435 pp.

de Bivort B. L., Clouse R. M. \& G. Giribet. 2010. A morphometrics-based phylogeny of the temperate Gondwanan mite harvestmen (Opiliones, Cyphophthalmi, Pettalidae). Journal of Zoological Systematics and Evolutionary Research, doi: 10.1111/j.1439-0469.2009.00562.x.

Calle D. A., Quiñónez M., Erazo H. \& N. Jaramillo. 2002. Morphometric discrimination of females of five species of Anopheles of the Subgenus Nyssorhynchus from Southern and Northwest Colombia. Memorias do Instituto Oswaldo Cruz, 97(8): 1191-1195.

Calle D. A., Quiñónez M., Erazo H. \& N. Jaramillo. 2008. Discriminación por morfometría geométrica de once especies de Anopheles (Nyssorhynchus) presentes en Colombia. Biomedica, 28: 371-385.

Camul R. \& P. D. Polly. 2005. Phylogenetic and environmental components of morphological variation: Skull, mandible, and molar shape in Marmots (Marmota, Rodentia). Evolution, 59(11): 2460-2472.

Carcavallo R. U., Curto de Casas S. I., Sherlock I. A., Girón I. G., Jurberg J., Galvão C., Mena Segura C. A. \& F. Noireau. 1999. Geographical distribution and alti-latitudinal dispersion. In: Carcavallo, R. U., Girón, I. G., Jurberg, J., Lent, H. (Eds.), Atlas of Chagas Disease Vectors in the Americas, vol. 3. Editora FIOCRUZ, Rio de Janeiro, pp. 747-792.

Carcavallo R. U., Jurberg J., Lent H., Noireau F. \& C. Galvão. 2000. Phylogeny of the Triatominae (Hemiptera:Reduviidae) proposals for taxonomic arrangements. Entomol Vect 7 (Suppl.1): 1-99.

Catalá S. 1997. Antennal sensilla of Triatominae Hemiptera, Reduviidae. A comparative study of five genera. International Journal of Insect Morphology and Embriology, 26: 67-73.

Catalano S., Goloboff P. A. \& N. Giannini. 2010. Phylogenetic morphometrics (I): the use of landmark data in a phylogenetic framework. Cladistics, 26: 11-20.

Cova-García P. \& O. Suarez. 1959. Estudio de los triatominos de Venezuela. Publ. Dir. de Malariología. Tip. Vargas S.A. Caracas, Venezuela, 209 pp.

Dujardin J. P., Garcia-Zapata M. T., Jurberg J., Roelants P., Cardozo L., Panzera F., Dias J. C. P. \& C. J. Schofield. 1991. Which species of Rhodnius is invading houses in Brazil. Transactions of the Royal Society of Tropical Medicine and Hygiene, 85: 679-680.

Dujardin J. P., Bermúdez H., Casini C., Schofield C. J. \& M. Tibayrenc. 1997. Metric differences between sylvatic and domestic Triatoma infestans (Hemiptera: Reduviidae) in Bolivia. Journal of Medical Entomology, 34: 544-551.

Dujardin J. P., Forgues G., Torrez M., Martinez E., Cordoba C. \& A. Gianella. 1998. Morphometrics of domestic Panstrongylus rufotuberculatus in Bolivia. Annals of Tropical Medicine and Parasitology, 92: 219-228.

Dujardin J. P., Chavez T., Moreno J. M., Machane M., Noireau F. \& C. J. Schofield. 1999. Comparison of isoenzyme electrophoresis and morphometric analysis for phylogenetic reconstruction of the Rhodniini (Hemiptera: Reduviidae: Triatominae). Journal of Medical Entomology, 36: 653-659.

Falconer D. S. 1981. Introduction to quantitative genetics. 4th ed. Prentice Hall, England. 300pp.

Farris J. 1970. Methods for computing Wagner trees. Systematic Zoology, 19: 83-92.

Farris J. A., Albert V. A., Källersjö M., Lipscomb D. \& A. G. Kluge. 1996. Parsimony jackknifing outperforms neighbor-joining. Cladistics, 12: 99-124.

Felsenstein J. 1985. Confidence limits on phylogenies: an approach using the bootstrap. Evolution, 39: 783-791. 
Felsenstein J. 2002. Quantitative characters, phylogenies, and morphometrics. In: Macleod N. \& P. L. Forey (Eds.), Morphology, Shape and Phylogeny. Systematics Association Special Volume Series 64. Taylor and Francis, London, p 27-44.

Feliciangeli M. D., Sanchez-Martin, M., Marrero R., Davies C. \& J. P. Dujardin. 2007. Morphometric evidence for a possible role of Rhodnius prolixus from palm trees in house re-infestation in the State of Barinas (Venezuela). Acta Tropica, 101: 169-177.

Galvão C., Carcavallo R. U., Rocha D. S. \& J. Jurberg. 2003. A checklist of the current valid species of the subfamily Triatominae Jeannel, 1919 (Hemiptera, Reduviidae) and their geographical distribution, with nomenclatural and taxonomic notes. Zootaxa, 202: 1-36.

Goloboff P. A. 1993. Character optimization and calculation of tree lengths. Cladistics, 9: 433-436.

Goloboff P. A., Mattoni C. I. \& A. S. Quinteros. 2006. Continuous characters analyzed as such. Cladistics, 22: 1-13.

Goloboff P. A., Farris J. \& K. Nixon. 2008. TNT, a free program for phylogenetic analysis. Cladistics, 24: 774-786.

Goloboff P. A. \& S.A. Catalano. 2010. Phylogenetic morphometrics (II): algorithms for landmark optimization. Cladistics, 26: 1-10.

Goncalves L. 2008. Morfometria geométrica de Psammolestes arthuri (Pinto, 1926) (Hemiptera, Reduviidae) del estado Guarico, Venezuela. Trabajo de Grado. Facultad de Ciencias y Tecnología. Universidad de Carabobo, 98 pp.

Gonzalez-José R., Escapa I., Neves W. A., Cúneo R. \& H. M. Pucciarelli. 2008. Cladistic analysis of continuous modularized traits provides phylogenetic signals in Homo evolution. Nature 6891: 1-5.

Guerrero, J. A., De Luna E. \& C. Sanchez-Hernández. 2003. Morphometrics in the quantification of character state identity for the assessment of primary homology: an analysis of character variation of the genus Artibeus (Chiroptera: Phyllostomidae). Biological Journal of the Linnean Society, 80: 45-55.

Gurgel-Gonçalves R. \& R. Bandeira. 2009. Analysis of the geographical distribution of Psammolestes Bergroth (Hemiptera: Hereroptera: Reduvidae: Triatominae) in South America, with new records of Psammolestes tertius Lent \& Jurberg. Zootaxa, 41-48.

Harry M. 1993a. Isozymic data question the specific status of some blood-sucking bugs of the genus Rhodnius, vectors of Chagas disease. Transactions of the Royal Society of Tropical Medicine and Hygiene, 87: 492-493.

Harry M. 1993b. Use of the median process of the pygophore in the identification of Rhodnius nasutus, $R$. neglectus, $R$. prolixus and $R$. robustus (Hemiptera: Reduviidae). Annals of Tropical Medicine and Parasitology, 87: 277-282.

Harry M., Galindez I. \& M. L. Cariou. 1992. Isozyme variability and differentiation between Rhodnius prolixus, $R$. robustus and $R$. pictipes, vectors of Chagas disease in Venezuela. Medical Veterinary Entomology, 6: 37-43.

Hypša V., Tietz D., Zrzavý J., Rego R. M. O., Galvão C. \& J. Jurberg. 2002. Phylogeny and biogeography of Triatominae (Hemiptera: Reduviidae): Molecular evidence of a New World origin of the Asiatic clade. Molecular Phylogenetics and Evolution, 23: 447-457.

Jaramillo N., Castillo D. \& M. Wolff. 2002. Geometric Morphometric Differences between Panstrongylus geniculatus from Field and Laboratory. Memorias do Instituto Oswaldo Cruz, 97: 667-673.

Lehmann P., Ordoñez R., Ojeda-Baranda R., Mendez de Lira J., Hidalgo-Sosa L., Monroy C. \& J. M. Ransey. 2005. Morphometric analysis of Triatoma dimidiata populations (Reduviidae: Triatominae) from Mexico and Northern Guatemala. Memorias do Instituto Oswaldo Cruz, 100: 477-482. 
Lent H. \& J. Jurberg. (1965) O gênero Psammolestes Bergroth, 1911, com um estudo sôbre a genitália das espécies (Hemiptera, Reduviidae, Triatominae). Revista Brasileira de Biología, 25: 349-376.

Lent H. \& P. Wygodzinsky. 1979. Revision of the Triatominae (Hemiptera: Reduviidae) and their significance as vectors of Chagas disease. Bulletin of the American Museum of Natural History, 163: $123-520$.

Lyman D. F., Monteiro F. A., Escalante A. A., Cordon-Rosales C., Wesson D. M., Dujardin J. P. \& C. B. Beard. 1999. Mitochondrial DNA sequence variation among triatomine vectors of Chagas disease. American Journal of Tropical Medicine and Hygiene, 60: 377-386.

Longa A. \& J. V. Scorza. 2005. Acrocomia aculeata (Palmae), hábitat silvestre de Rhodnius robustus en el Estado Trujillo, Venezuela. Parasitología Latinoamericana, 60: 17-24.

Matias A., de la Riva J. X., Torrez M. \& J. P. Dujardin. 2001. Rhodnius robustus in Bolivia Identified by its Wings. Memorias do Instituto Oswaldo Cruz, 96: 947-950.

Monroy C. Bustamante D. M., Rodas A., Rosales-Mejía M. \& Y. Tabaru. 2003. Geographic distribution and morphometric differentiation of Triatoma nitida Usinger 1939 (Hemiptera: Reduviidae: Triatominae) in Guatemala. Memorias do Instituto Oswaldo Cruz, 98: 37-43.

Monteiro F. A., Wesson D. M., Dotson E. M., Schofield C. \& C. B. Beard. 2000. Phylogeny and Molecular taxonomy of the Rhodniini derived from mitochondrial and nuclear DNA sequences. American Journal of Tropical Medicine and Hygiene, 62: 460-465.

Norman G. R. \& D. L. Striner. 1994. Bioestadística. Madrid: Editorial Harcourt S.A.

Osuna E. 1984. Notas sobre Triatominae (Hemiptera: Reduviidae) del Parque Nacional "Cerro de la Neblina” Territorio Federal Amazonas, Venezuela. Boletín de la Dirección de Malariología y Saneamiento Ambiental, 24: 45-46.

Osuna E. \& J. M. Ayala. 1993. Belminus pittieri, nueva especie de Bolboderini (Triatominae: Reduviidae: Heteroptera). Boletín Entomología Venezolana, 8: 147-150

Ribeiro J. M., Schneider M., Isaias T., Jurberg J., Galvão C. \& J. A. Guimarães. 1998. Role of salivary antihemostatic components in blood feeding by triatomine bugs (Heteroptera). Journal of Medical Entomology, 35: 599-610.

Rubio-Pallis Y. 1998. Caracterización morfométrica de poblaciones de Anopheles (Nyssorhynchus) darlingi del sur de Venezuela. Boletín Entomología Venezolana,13: 141-172.

Rohlf F. J. 2003. TpsRelw, Program provides a low dimensional approximation (via a principal components analysis) to the tangent space approximation of shape space, version 1.37. Department of Ecology and Evolution, State University of New York at Stony Brook. Disponible en: http://life.bio. sunysb.edu/morph/index.html.

Rohlf F. J. 2007. tpsDig, Program for Digitalizing morphologic landmark and outlines for Geometric Morphometric Analyses, version 2.04. Department of Ecology and Evolution, State University of New York at Stony Brook. Disponible en: http://ife.bio.sunysb.edu/morph/index.html.

Rohlf F. J. \& F. Marcus. 1993. A Revolution in Morphometrics. Trends in Ecology and Evolution, 8 (4): 129-132.

Schofield C. J. 1988. Biosystematics of the Triatominae, In: Service M.W. (Ed.). Biosystematics of Haematophagous Insects. Oxford: Clarendon Press, pp. 281-312.

Schofield C. J. 1994. Triatominae-Biology \& Control. Eurocommunica Publications, UK. 80 pp.

Schofield C. J. 1996. Biosystematics and adaptive trends in Triatominae. In: C.J. Schofield, J.P. Dujardin, J. Jurberg (Eds.). Proceedings of the International Workshop on Population Genetics and Control of Triatominae, Santo Domingo de los Colorados, Ecuador. INDRE Mexico City, pp. 45-50.

Schofield C. J. \& J. P. Dujardin. 1999. Theories of the evolution of Rhodnius. Actualidades Biológicas, 21: 183-197. 
Schofield C. J. \& C. Galvão. 2009. Classification, Evolution, and species groups within the Triatominae. Acta Tropica, 110: (2-3): 88-100.

Sheets H. D. 2005a. CoordGen6, Coordinate Generation program for calculating shape coordinates. Disponible en: http://www3.canisius.edu/ sheets/morphsoft.html

Sheets H.D. 2005b. CVAGen6, Canonical Variates Analysis program for the analysis of shape, based on partial warp scores. Disponible en: http://www3.canisius.edu/ sheets/morphsoft.html.

Soto-Vivas A., Rodríguez C., Bonfante R. \& E. Aldana. 2007. Morfometría geométrica de Triatoma maculata (Erichson, 1848) de ambientes doméstico y peridoméstico, estado Lara, Venezuela. Boletín de Malariologia y Salud Ambiental, 47: 231-235.

Soto-Vivas A. 2009. Clave pictórica triatóminos (Hemiptera: Triatominae) de Venezuela. Boletín de Malariologia y Salud Ambiental, 49: 259-274.

Tartarotti E., Azeredo-Oliveira M. T. V. \& C. R. Ceron. 2006. Phylogenetic approach to the study of triatomines (Triatominae, Heteroptera). Brazilian Journal of Biology, 66: 703-708.

Torrealba J. W., Tonn R. J. \& R. U. Carcavallo. 1985. Venezuela. In: R. U. Carcavallo, J. E. Rabinovich, R. J. Tonn (Eds.), Factores Biológicos y Ecológicos en la Enfermedad de Chagas. Centro Panamericano de Ecología Humana y Salud, Argentina.

WHO. 1990. Tropical Diseases 1990. TDR/CTD/HH20.1. Geneva: WHO, 26 pp.

World Bank. 1993. World Development Report 1993. Investing in Health. Oxford University Press, New York.

Yurtas H., Alten B. \& A. N. Aytekin. 2005. Variability in natural populations of Anopheles sacharovi (Diptera: Culicidae) from southeast Anatolia, revealed by morphometric and allozymic analyses. Journal of Vector Ecology, 30(2): 206-212.

Zelditch M., Swiderski D. L., Sheets H. D. \& W. L. Fink. 2004. Geometric morphometrics for biologists: a primer. Boston: Elsevier Academic Press. 444 pp. 